\title{
AV Fistula Aneurysm
}

National Cancer Institute

\section{Source}

National Cancer Institute. AV Fistula Aneurysm. NCI Thesaurus. Code C114753.

A bulge in a weakened vessel wall segment of an arteriovenous fistula. 